\title{
The relationship between adolescents' perceived parental involvement, self-efficacy beliefs, and subjective well-being: a multiple mediator model
}

\begin{abstract}
The aim of this study was to examine the mediation roles of academic self-efficacy, social self-efficacy, and emotional self-efficacy on the relationships between parental involvement (i.e., paternal involvement and maternal involvement) and subjective well-being (i.e., positive affect, negative affect, and life satisfaction) in Malaysian adolescents. Participants were 802 Malaysian high school students from 14 public schools, with an age range of $15 \mathrm{I} 17$ years. Results of a multiple mediator model indicated that academic self-efficacy and social selfefficacy were unique mediators in the relationships between parental involvement (both paternal involvement and maternal involvement) and adolescent positive affect. Besides, academic self-efficacy was found to be the only unique mediator in the relationships between parental involvement and adolescent life satisfaction. Emotional self-efficacy did not uniquely mediate the relationships between parental involvement and adolescent positive affect and life satisfaction. None of the proposed mediators uniquely mediated the relationships between parental involvement and adolescent negative affect. This study suggests that paternal involvement is just as crucial to adolescent positive development as maternal involvement. In addition, this study also extends our insight into the specific roles of academic, social, and emotional self-efficacy in the relationship between parental involvement and the components of subjective well-being among adolescents.
\end{abstract}

Keyword: Parenting; Self-efficacy; Positive affect; Negative affect; Life satisfaction; Multiple mediator model 